\title{
Sexualidade no pós-parto: percepção das mulheres e atuação da enfermagem
}

Todo ser humano é formado por questões complexas de sexualidade, fazendo parte da vida, de forma significativa. A sexualidade é compreendida por fatores diversos e envolve questões biopsicossociais, estando diretamente relacionada com a identidade pessoal e humana, além de níveis de satisfação com a própria vida, evidenciando a complexibilidade de sua conceituação, pois, não se trata apenas de questões físicas e do prazer propriamente dito. Tem como objetivo em identificar a percepções das mulheres em relação à sexualidade no período do pós-parto e as ações de enfermagem. Este estudo propôs a realização de uma pesquisa de campo, de metodologia quantitativa, exploratório-descritiva, no qual os dados foram coletados a partir de um questionário semiestruturado desenvolvido pelas pesquisadoras, baseado em artigo sobre sexualidade no pós-parto. De acordo com a abordagem dos resultados, foi possível identificar que as mulheres que passam pelo puerpério, em muitas vezes tem dificuldades em retornar a ter relações sexuais e consideram que é possível voltar a ter uma vida sexual no período do pós-parto. Foi observado nesse estudo que a grande maioria das mulheres não teve receio de retornar as práticas sexuais no pós-parto apesar de que muitas não tiveram o retorno do desejo sexual de forma imediata, foi algo que demorou um tempo, variando esse tempo de mulher para mulher no grupo que foi entrevistado. Essas mulheres também voltaram a ter relações sexuais apesar dos desconfortos que tiveram na primeira relação no pós-parto, isso mostra que os desconfortos podem ser contornados, e se faz necessário o diálogo da mulher para com o seu parceiro nesse momento.

Palavras-chave: Puerpério; Mulher; Enfermagem.

\section{Postpartum sexuality: women's perception and nursing performance}

Every human being is formed by complex issues of sexuality, being part of life, in a significant way. Sexuality is understood by several factors and involves biopsychosocial issues, being directly related to personal and human identity, in addition to levels of satisfaction with life itself, showing the complexity of its conceptualization, as it is not only about physical issues and the pleasure itself. It aims to identify women's perceptions of sexuality in the postpartum period and nursing actions. This study proposed conducting a field research, with a quantitative, exploratory-descriptive methodology, in which the data were collected from a semi-structured questionnaire developed by the researchers, based on an article on sexuality in the postpartum period. According to the approach of the results, it was possible to identify that women who go through the puerperium often have difficulties in returning to have sexual relations and consider that it is possible to have a sexual life again in the postpartum period. It was observed in this study that the great majority of women were not afraid to return to sexual practices in the postpartum period, although many did not have the return of sexual desire immediately, it was something that took some time, varying this time as a woman for women, in the group that was interviewed. These women also had sex again despite the discomfort they had in the first postpartum relationship, this shows that the discomfort can be overcome, and it is necessary for the woman to dialogue with her partner at that time.

Keywords: Puerperium; Women; Nursing.

Reviewed anonymously in the process of blind peer.

Fabrine Abreu de Sousa Faculdade Guaraí, Brasi fabrine_abreu@hotmail.com

\section{Juliana Monteiro Pereira}

Faculdade Guaraí, Brasil

julianamonteira@hotmail.com

Glaucya Wanderley Santos Markus

Faculdade Guaraí, Brasi

http://lattes.cnpq.br/5568510365985231

glaucyamarkus@outlook.com
Karla Camila Correia da Silva

Faculdade Guaraí, Brasil

http://lattes.cnpq.br/1981447087125364

karlacamilac@yahoo.com.br

Giullia Bianca Ferraciolli do Couto

Faculdade Guaraí, Brasil

http://lattes.cnpq.br/0109560699727614

giulliabianca@hotmail.com

Reobbe Aguiar Pereira

Faculdade Guaraí, Brasil

http://lattes.cnpq.br/7447115724350334

enfreobbe@gmail.com
Adriana Keila Dias

Faculdade Guaraí, Brasil

http://lattes.cnpq.br/2128882976477548

drianakeiladias@hotmail.com

Juliane Marcelino dos Santos

Faculdade Guaraí, Brasi

http://lattes.cnpq.br/8751832120632358

julianemarcelino@hotmail.com
Referencing this:

SOUSA, F. A.; PEREIRA, J. M.; MARKUS, G. W. S.; SILVA, K. C. C.; COUTO, G. B. F.; PEREIRA, R. A.; DIAS, A. K.; SANTOS, J. M.

Sexualidade no pós-parto: percepção das mulheres e atuação da enfermagem. Scire Salutis, v.11, n.3, p.61-68, 2021. DOI: http://doi.org/10.6008/CBPC2236-9600.2021.003.0009 


\section{INTRODUÇÃO}

Todo ser humano é formado por questões complexas de sexualidade, fazendo parte da vida, de forma significativa. A sexualidade é compreendida por fatores diversos e envolve questões biopsicossociais, estando diretamente relacionada com a identidade pessoal e humana, além de níveis de satisfação com a própria vida, evidenciando a complexibilidade de sua conceituação, pois, não se trata apenas de questões físicas e do prazer propriamente dito (SOUSA, 2019). A sexualidade quando vivenciada no contexto da gravidez e pós-parto ganha outros significados e sentidos, tanto para a mulher como também para o parceiro. O pós-parto trata-se de um período de grande pluralidade sexual, trazendo novas realidades para a vida da mulher de forma especial. Nesse período várias vertentes são importantes para a manutenção da sexualidade, sendo essas culturais, fisiológicas, psicológicas e a relação intrínseca entra mulher e parceiro (OLIVEIRA et al., 2015).

Segundo pesquisas $53 \%$ das mulheres apresentam problemas com a sexualidade no período do puerpério, sendo os mais comuns: a falta de vontade sexual $(34,6 \%)$ falta de sensação de prazer completo $(29,3 \%)$ dores em regiões sexuais $(21 \%)$, além de problemas com vaidade e motivação pessoal, que também fazem parte da sexualidade (VETTORAZZI et al., 2012). É chamado de pós-parto o período que compreende a fase onde a mulher passa por alterações físicas e psíquicas até que retorne ao estado anterior à sua gravidez. Esse período se inicia logo após, logo após o nascimento do bebê. Durante esta fase, o corpo da puérpera está em processo de estabilização, voltando ao que era antes da gravidez (VIEIRA et al., 2016).

Nesse momento diversas mudanças aparecem na vida da mulher. As alterações psicológicas e fisiológicas são as mais evidentes, isso porque nesse momento com a chegada de um novo ser, a rotina e os conceitos podem ser transformados. Com essa situação, várias mulheres passam dificuldades para a adaptação, gerando impactos psicológicos que podem influenciar diretamente na vivência da sexualidade pessoal e na vida do parceiro (BRASIL, 2016). Desta forma, a enfermagem assumiu papel importante na manutenção da sexualidade no pós-parto, isso porque o enfermeiro tem vários instrumentos que the auxiliam, tanto as consultas de enfermagem na atenção primariam como também à educação em saúde. A enfermagem tem uma assistência holística e humanizada, sendo forte aliada no enfrentamento das queixas sexuais puerperais, e sendo uma válvula de escape entre a puérpera e sua família (BRASIL, 2016).

Diante da percepção do problema, surge a seguinte problemática: Quais os principais problemas de sexualidade no período puerperal? De que forma enfermagem pode influenciar na superação dessas queixas? Essa pesquisa se justifica na necessidade de se intensificar os estudos em relação à sexualidade no puerpério, tendo em vista que essa temática é muito burlada e alguns profissionais não levam a sério esse aspecto. A sexualidade influencia diretamente nas atividades gerais da mulher e da família, enfatizando sua importância e demonstrando como os serviços de saúde precisam ter atenção quanto a ela. Diante disso este artigo tem como objetivo identificar a percepções das mulheres em relação à sexualidade no período do pós-parto e as ações de enfermagem, descrever as principais queixas sobre a sexualidade no período pós-parto, caracterizar a percepção da mulher em relação à sexualidade no pós-parto e descrever a 
assistência de enfermagem as mulheres no pós-parto em relação à sexualidade.

\section{METODOLOGIA}

Este estudo propôs a realização de uma pesquisa de campo, de metodologia quantitativa, exploratório-descritiva, no qual os dados foram coletados a partir de um questionário semiestruturado desenvolvido pelas pesquisadoras, baseado em artigo sobre sexualidade no pós-parto. A pesquisa foi realizada com gestantes que realizaram o pré-natal nas UBS, no município de Pedro Afonso- TO e Bom Jesus-TO. A população do estudo foi composta por 15 gestantes da Unidade Básica de Saúde Tenente Salustiano e 15 gestantes Unidade Básica de Saúde Francisco Pereira da Rocha.

OS dados foram coletados do dia 15 de setembro ao dia 28 de outubro de 2020, no horário de funcionamento das unidades básicas de saúde, a amostra foi de 30 gestantes. Foram utilizados como critérios de inclusão, mulheres que fazem acompanhamento e consultas de enfermagem nas Unidades Básicas de Saúde de Pedro Afonso e de Bom Jesus do Tocantins, mulheres com até é 40 dias pós-parto, que ainda esteja com o seu companheiro e que tenha no mínimo 18 anos de idade e que aceitaram participar da pesquisa mediante a assinatura do termo de Consentimento Livre e Esclarecido (TCLE), e como critérios de exclusão, Mulheres que não são acompanhadas pela Estratégia Saúde da Família, que tenham mais de 40 dias pós-parto, que tenha mais de 6 meses pós-parto, que não tenha um companheiro e que seja menor de 18 anos. A pesquisa foi realizada com autorização pelo secretário de saúde do município de Pedro Afonso - TO e de Bom Jesus-TO, levando-se em consideração os aspectos éticos em pesquisas que envolvem seres humanos, conforme descrito na Resolução n 466/2012, do Conselho Nacional de Saúde (CNS), que regulamenta a pesquisa envolvendo seres humanos. A pesquisa obteve a aprovação do Comitê de Ética em Pesquisa do Centro Universitário Tocantinense Presidente Antônio Carlos - UNITPAC, sob o Parecer № 4.574.961.

\section{RESULTADOS E DISCUSSÃO}

Os resultados encontrados foram sistematizados. Estruturados em gráficos e tabelas e analisados diante a literatura. Os gráficos serão identificados segundo suas temáticas, e as perguntas que estiveram no instrumento de coleta, serão explicitadas nas análises para o entendimento do contexto.

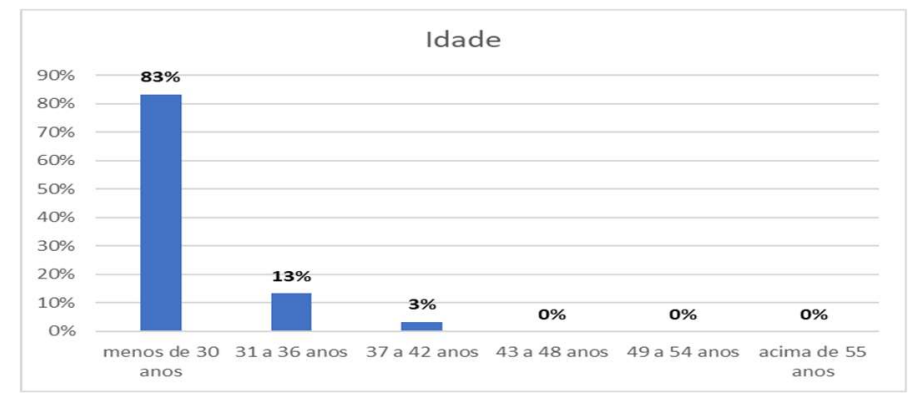

Gráfico 1: Perfil das mulheres por faixa etária

O gráfico 01, apresenta a faixa etária das mulheres que participaram dessa pesquisa, observar se que $83 \%$ têm menos que 30 anos, 13\% têm entre 31 e 36 anos e que 3\% têm entre 37 a 42 anos. Pode se 
observar que o maior índice de mulheres participantes da pesquisa, compreende mulheres com menos de 30 anos, espera-se que essas mulheres tenham sido orientadas a respeito da importância de se esperar pelo menos os 40 dias pós-parto para o início das relações sexuais, em vista de que isso é uma forma de cuidar da sua saúde sexual, pois no parto o corpo passa por severas mudanças e o mesmo precisa de um tempo para que volte ao normal, que seria o estado anterior a gestação.

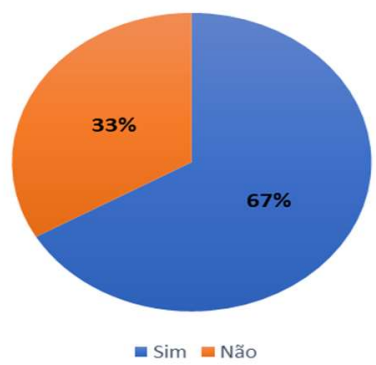

Gráfico 2: Mudanças observadas no período pós-parto

$\mathrm{Na}$ análise do gráfico 02 , pode se observar que $67 \%$ das entrevistadas, observaram mudanças significativas em seus corpos no período pós-parto. Essas mudanças se relacionam ao físico de cada mulher, uma vez que o corpo feminino passa por muitas mudanças neste período.

As mudanças que ocorrem no corpo da mulher nesse período, podem tanto ser pequenas, quanto grandes, essas mudanças variam de acordo com o corpo de cada mulher. Tudo esse contexto de modificações que acontecem, tem grandes implicações no dia a dia de cada mulher, pois muitas passam a não se sentir mais bonitas ou desejáveis por seus parceiros, uma vez que os seus corpos não são mais mesmos, o tempo para cuidar de si mesma também diminui, e tudo isso vem de impacto com a sexualidade feminina (CASTIGLIONI et al., 2020).

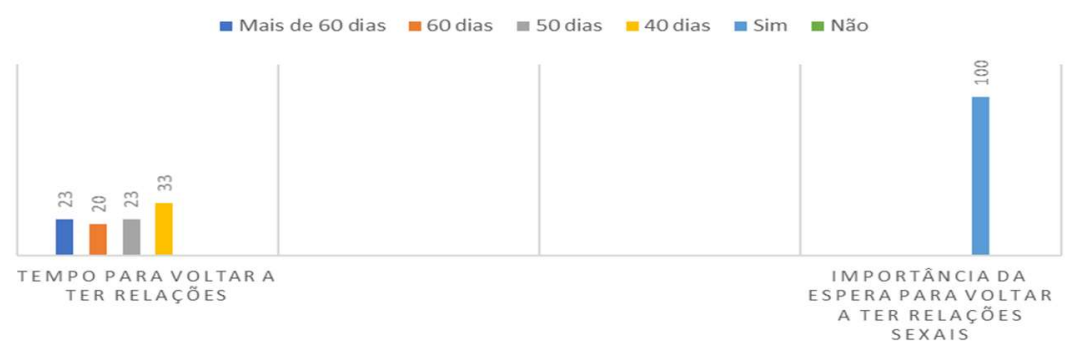

Gráfico 3: Tempo de espera para voltar a ter relações e Importância da espera para voltar a ter relações sexuais

Analisando o gráfico 03, pode observar-se que 33\% das entrevistadas esperaram 40 dias pós-parto para voltar a ter relações sexuais, isso mostra que que esperaram o tempo indicado mínimo para voltar a ter relações e também que $100 \%$ das entrevistadas, consideram importante esperar o período mínimo de 40 dias para voltar a ter relações sexuais. Essa afirmação por parte das entrevistadas, demostra o cuidado que as mesmas almejam ter em relação aos seus corpos. O período do resguardo é o momento que traz muitas manifestações em relação ao cuidado da mulher com o seu corpo, com o intuito de se reestabelecer, pois além de mãe, ela ainda e mulher, e o ser mulher ainda e importante para o seu estado, físico e emocional. A espera de 40 dias para voltar a ter relações sexuais, traz consigo uma grande importância, uma vez que o corpo da mulher precisa de tempo para se recuperar das alterações ocorridas 
durante o parto. É necessário que a mulher entre em acordo com o seu parceiro, para que ambos possam aguardar o tempo mínimo de espera no resguardo (CASTIGLIONI et al., 2020).

Trabalhando com o contexto da importância dessa espera, pode se tomar como entendimento que a mulher e o seu parceiro, devem ter paciência um com o outro, quando se tratar das relações sexuais, e ambos podem juntos procurar outras formas de sentir prazer, que não tenham haver especificamente com o ato sexual. Deve-se estar de olho também na volta do desejo sexual da mulher, sendo que muitas mulheres demoram a ter desejo sexual novamente (ROSALINO, 2020).

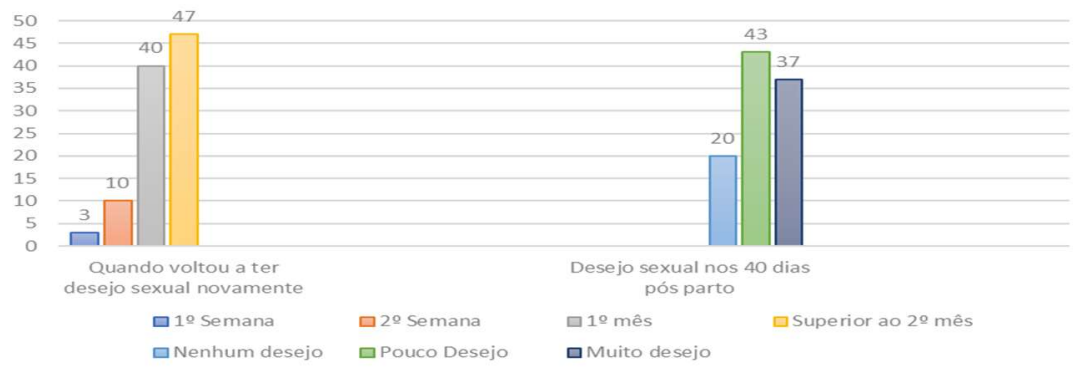

Gráfico 4: O retorno do desejo sexual e a Presença do desejo sexual nos 40 dias pós-parto

Analisando o gráfico 04 , nota-se que $47 \%$ das entrevistadas só voltaram a ter desejo sexual novamente após o segundo mês pós-parto. Esse índice tão elevado para a volta do desejo sexual se deve, ao fato de que a mulher perde muito do seu desejo sexual nesse período em virtude das alterações hormonais que a mesma passa e também vemos que $43 \%$ das entrevistadas responderam que tiveram pouco desejo sexual após o tempo de espera para o retorno da prática sexual.

Neste momento em que muitas mulheres se encontram sem nenhum tipo de desejo sexual, cabe a elas conversarem com os seus parceiros, para que juntos, eles possam encontram maneiras de sentir prazer de outras formas, auxiliando assim o retorno do desejo sexual dessa mulher. As mulheres também podem estar procurando profissionais que sejam especializados, para que possam estar auxiliando essas mulheres em melhores formas de ter o retorno da libido (CABRAL et al., 2020).

Com base em estudos, esse dado, se deve ao fator de que após o nascimento e as inúmeras mudanças pelas quais o corpo da mulher passa, a diminuição da libido acaba acontecendo com a grande maioria das mulheres que estão na fase do pós-parto. Com o nascimento do bebê, há uma queda significativa de hormônio no corpo da mulher, uma vez que ele não precisa mais de uma carga tão alta de hormônios correntes no sangue, pois o bebê já nasceu (CABRAL et al., 2020).

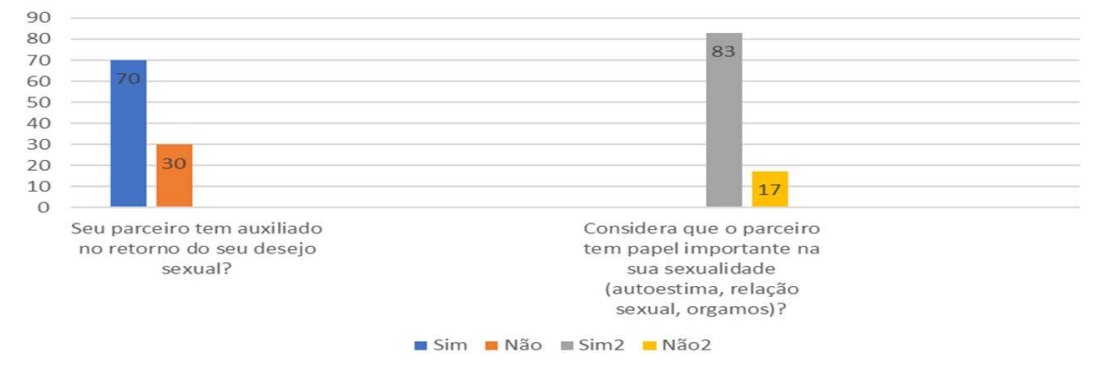

Gráfico 5: Auxílio do parceiro no retorno do desejo sexual e o papel do parceiro na sexualidade

Olhando no gráfico 5 temos uma grande maioria de $70 \%$ das mulheres afirmando que sim, seus 
parceiros tem ajudado e auxiliado no retorno de seu desejo sexual e libido, essa resposta positiva se faz importante uma vez que esse auxilio tem grande impacto no retorno desse desejo sexual e vemos que a maioria das entrevistadas responde sim para o questionamento a respeito da importância do papel do parceiro em sua sexualidade.

Quando a mulher tem um apoio do seu parceiro, na questão do retorno do desejo sexual e libido ela vem a se sentir mais confiante no que está fazendo, pois o parceiro em muitas vezes está com ela há muito tempo, e sendo assim já existem laços de confiança entre os dois, e isso facilita o processo (ROSALINO, 2020). O parceiro deve estar em constante diálogo com a mulher, para conseguir entender os seus medos e anseios nesse momento, e dessa forma ambos buscarem a melhor forma de passar por esse momento. E quando encontrarem uma forma, ambos trabalharem juntos para que consigam esse objetivo, tendo em mente que é um processo gradual, que varia de pessoa para pessoa e que cada mulher leva o seu tempo para que isso ocorra (ROSALINO, 2020).

Essa resposta afirmativa nos demonstra que a conexão das mulheres com os seus parceiros produz uma onda de confiança, onde a forma como o parceiro trata a mulher e se relaciona com ela, vai estar intimamente ligada à como ambos vão interpretar a sua sexualidade. A mulher que se sente segura para isso, tem uma maior facilidade de voltar a ter relações no período pós-parto, uma vez que este retorno em muitos casos e um momento difícil para muitas mulheres (JESUS et al., 2017).

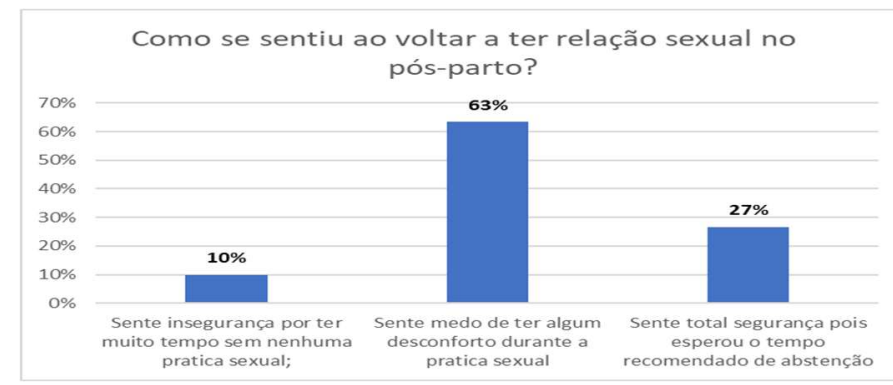

Gráfico 6: O retorno da relação sexual no pós-parto

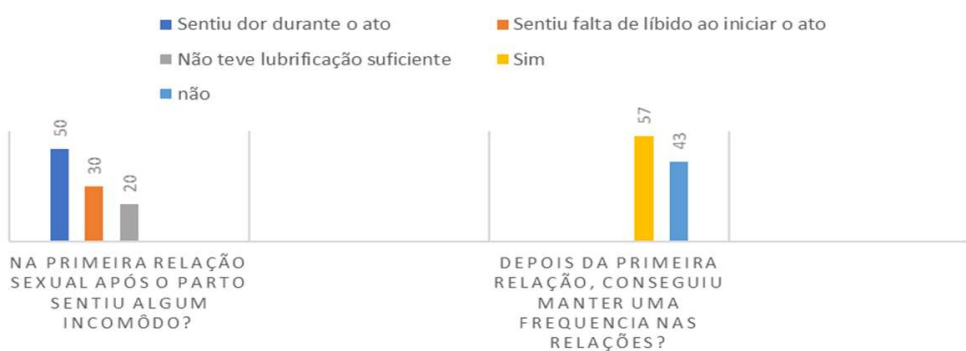

Gráfico 7: A primeira relação sexual no pós-parto e a frequência das relações sexuais no pós-parto.

Como está exposto no gráfico 9, 63\% das mulheres que foram entrevistadas, afirmaram sentir medo de ter algum tipo de desconforto durante a prática sexual, uma vez que elas passaram por um período de tempo relativo antes de iniciar a prática sexual novamente. Este medo deve do retorno à prática sexual, deve ser observado como medo do desconhecido, uma vez que a mulher tinha ciência de como era ter relações sexuais antes do parto, e após o parto seu corpo passou por muitas mudanças, então e algo desconhecido para elas. Essas mulheres nesse momento estão começando a retornar as atividades normais 
que faziam antes de ter o bebê e isso implica em tempo e paciência para que as mesmas consigam fazer isso de forma mais tranquila possível (ZALDIVAR, 2020).

O gráfico 07 , demonstra que sim, as mulheres sentiram desconforto durante o ato sexual, no período do pós-parto, isso deve tanto ao medo que muitas sentem desse retorno, quanto à quantidade de tempo que elas passaram sem ter relação sexual com os seus parceiros e demostra a frequência que as mulheres conseguiram manter nas relações sexuais no pós-parto.

O fato de ter algum desconforto durante a primeira relação sexual no pós-parto, pode se relacionar a diversos fatores, podemos listar as mudanças corporais que ocorreram com a mulher nesse período, pois elas influenciam muito como vai ocorrer essa primeira relação. Ainda observando essa primeira relação, temos também o fator de que muitas perdem a libido e consequentemente tem falta de lubrificação, fazendo assim com que essa primeira relação seja mais desconfortável para as mulheres (LEITE et al., 2019). Após a primeira relação sexual no pós-parto, muitas mulheres conseguem manter uma frequência na relação, porém isso não é regra, uma vez que cada mulher reage de uma forma nesse período, mediante as mudanças que cada uma delas passou. Neste momento a mulher deve observar as mudanças que aconteceram em seu corpo, os incômodos que a mesma sentiu na primeira relação, e juntamente com o seu parceiro encontrar meios para que consigam manter uma frequência de relações sexuais de uma forma que seja bom pra ambos.

A mulher deve conseguir se sentir confortável com durante as relações sexuais, pois neste momento de transição esse ato pode ajudar a mulher, a se sentir um pouco como ela era antes desse período. Este momento tem sua importância uma vez que as relações sexuais não têm a ver somente com o prazer durante o ato, mais uma prova de que a mulher pode retornar a fazer as atividades que fazia antes de ter o bebê (LEITE et al., 2019).

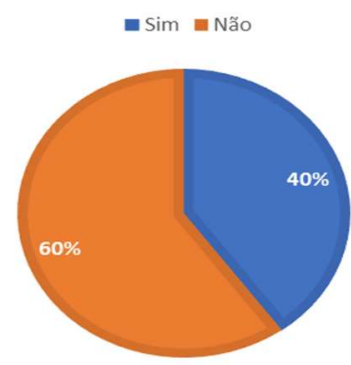

Gráfico 8: O retorno das relações sexuais com 40 dias pós-parto

É recomendado pelos profissionais da saúde que a mulher somente retorne a ter relações sexuais após o prazo de 40 dias, isso para que o corpo da mulher retorne ao que era antes do parto e que também se recupere do parto, momento esse em que acontecem muitas modificações no corpo feminino. É normal que muitas mulheres tenham dúvidas a respeito de ter ou não relações sexuais após esse prazo de 40 dias, pois muitas podem ainda se sentir inseguras sobre tal ato ou mesmo não confiarem que seja o momento certo de fazer isso. Algumas mulheres voltam a ter relações sexuais antes desse período pois entendem que pra elas é seguro voltar a pratica sexual, apesar de que não seja o recomendado, mas cabe à mulher seguir a orientação dos profissionais da saúde que a acompanharam no seu período da gestação, para que 
esse retorno seja o mais seguro possível (JESUS et al., 2017).

\section{CONCLUSÕES}

Foi observado nesse estudo que a grande maioria das mulheres não teve receio de retornar as práticas sexuais no pós-parto, apesar de que muitas não tiveram o retorno do desejo sexual de forma imediata foi algo que demorou um tempo, variando esse tempo de mulher para mulher, no grupo que foi entrevistado. Essas mulheres também voltaram a ter relações sexuais apesar dos desconfortos que tiveram na primeira relação no pós-parto, isso mostra que os desconfortos podem ser contornados, e se faz necessário o diálogo da mulher para com o seu parceiro nesse momento.

Faz-se necessária a melhor orientação dessas mulheres, tendo em vista que muitas possuem algum desconhecimento de como pode ser esse retorno às práticas sexuais, onde essas mulheres em alguns casos não esperaram o tempo mínimo recomendado para o retorno das relações sexuais. Cabe aos profissionais da saúde, orientar essas mulheres da melhor forma possível durante o período gestacional, para que no pós-parto elas tenham uma experiência mais tranquila possível.

\section{REFERÊNCIAS}

BRASIL. Protocolos da atenção básica: saúde das Mulheres. Brasília: Ministério da Saúde, 2016.

CABRAL, J. C.; FRIGO, L. F.. Disfunção sexual em mulheres no período pós-parto: revisão da literatura. Pesquisa, Sociedade e Desenvolvimento, v.9, n.9, 2020. DOI: https://doi.org/10.33448/rsd-v9i9.6785

CASTIGLIONI, C. M.; CREMONESE, L.; PRATES, L. A.; SCHIMITH, M. D.; SEHNEM, G. D.; WILHELM, L. A.. Práticas de cuidado no puerpério desenvolvidas por enfermeiras em estratégias de saúde da família. Rev. Enferm. UFSM REUFSM, v.10, n.50, p.1-19, 2020.

GOMES, A. T.; SCALCO, C. P. S.; VARELA, D.. Função e disfunção sexual feminina durante o ciclo gravídicopuerperal: uma revisão da literatura. Revista Brasileira De Sexualidade Humana, v.30, n.1, 2020. DOI: https://doi.org/10.35919/rbsh.v30i1.69

JESUS, W. G.; AZEVEDO, V. M. G. O.. Sexualidade no puerpério: a visão do casal. Enfermagem Obstétrica, v.4 n.58, 2017.

KLIEMANN, B. S. R. A.. Sintomas de disfunções sexuais femininas após o período puerperal. Monografia (Graduação em Psicologia) - Universidade do Sul de Santa Catarina, Florianópolis, 2018.

LEITE, B.; RIGO, E.; MIRANDA, H. J. C.; BARAZZETTI, L. Prevalência de disfunções sexuais femininas: uma revisão bibliográfica. CONGRESSO DE PESQUISA E EXTENSÃO DO CENTRO UNIVERSITÁRIO DA SERRA GAÚCHA, 7. Anais. Centro Universitário da Serra Gaúcha, 2020.

MARAMBAIA, C. G.; VIEIRA, B. D. G.; ALVES, V. H.; RODRIGUES, D. P.; ALMEIDA, V. L. M.; CALVÃO, T. F.. A sexualidade da mulher no puerpério: reflexos da episiotomia. Cogitare enferm, v.25, 2020. DOI: http://dx.doi.org/10.5380/ce.v25i0.67195.

OLIVEIRA, G. F.. Discursos de mulheres sobre sexualidade na amamentação. Rev enferm UFPE on line, v.9, n.6, 2015.

OLIVEIRA, T. S.. Fatores relacionados à disfunção sexual no puerpério. Monografia (Graduação em Medicina) Universidade Federal de Sergipe, 2018.

ROSALINO, D. A. T.. Um olhar para a mulher e suas necessidades de saúde no período puerperal a partir da integralidade do cuidado. São Carlos, 2020.

SIQUEIRA, L. K. R.; MELO, M. C. P.; MORAIS, R. J. L.. Pósparto e sexualidade: perspectivas e ajustes maternos. Revista de Enfermagem da UFSM, v.9, p.58, 2019.

SOUSA, I. G. T.. Queixas relacionadas à sexualidade no puerpério: uma revisão de literatura. Palmas, 2019.

VETTORAZZI, J.. Sexualidade e puerpério: uma revisão da literatura. Rev HCPA, v.32, n.4, 2012.

VIEIRA, T. G.. Percepção de gestantes acerca da sexualidade e o papel do enfermeiro como educador na atenção primária à saúde. Rev Temas em saúde, v.16, n.2, p.258-820, João Pessoa, 2016.

ZALDIVAR, A. P.; PRATES, L. A.; PEREZ, R. V.; GOMES, N. S.; PILGER, C. H.. Vivências de casais acerca da participação do parceiro no puerpério. Research, Society and Development, v.9, n.7, 2020. DOI: http://dx.doi.org/10.33448/rsdv9i7.4510

A CBPC - Companhia Brasileira de Produção Científica (CNPJ: 11.221.422/0001-03) detém os direitos materiais desta publicação. Os direitos referem-se à publicação do trabalho em qualquer parte do mundo, incluindo os direitos às renovações, expansões e disseminaç̃oses da contribuição, bem como outros direitos subsidiários. Todos os trabalhos publicados eletronicamente poderão posteriormente ser publicados em coletâneas impressas sob coordenação da Sustenere Publishing, da Companhia Brasileira de Produção Científica e seus parceiros autorizados. Os (as) autores (as) preservam os direitos autorais, mas não têm permissão para a publicação da contribuição em outro meio, impresso ou digital, em português ou em tradução. 